\title{
The Combination of Cisplatinum and Doxorubicin Regressed Primary Osteosarcoma of the Breast in a PDOX Mouse Model
}

\author{
NATHANIEL F. WU ${ }^{1,2}$, JUN YAMAMOTO ${ }^{1,3}$, YUSUKE AOKI ${ }^{1,3}$, NORIYUKI MASAKI ${ }^{1,3}$, \\ CARISSA SAMONTE ${ }^{1}$, JUSTIN WU $\mathrm{JU}^{3,4}$, MICHAEL BOUVET $^{3}$ and ROBERT M. HOFFMAN ${ }^{1,3}$ \\ ${ }^{1}$ AntiCancer Inc, San Diego, CA, U.S.A.; \\ ${ }^{2}$ Department of Molecular and Cellular Biology, Harvard University, Cambridge, MA, U.S.A.; \\ ${ }^{3}$ Department of Surgery, University of California, San Diego, CA, U.S.A.; \\ ${ }^{4}$ Department of General Surgery, Kaiser Permanente San Diego Medical Center, San Diego, CA, U.S.A.
}

\begin{abstract}
Background/Aim: Primary osteosarcoma of the breast is an exceedingly-rare malignant tumor that shares histological characteristics with osteosarcoma of the bone. Since effective therapies have not yet been established, standard therapy for osteosarcoma of the bone was examined in the present study for efficacy against primary osteosarcoma of the breast in a patient-derived orthotopic xenograft (PDOX) nudemouse model. Materials and Methods: The PDOX mouse models were established by surgical implantation of the primary osteosarcoma of the breast specimen into the mammary gland of nude mice. Mice with tumors were randomized into four groups, each $n=4$ : control group; cisplatinum (CDDP)treatment group; doxorubicin (DOX)-treatment group; and $C D D P / D O X$-combination-treatment group. Mice were treated for twenty-one days, three weeks after implantation. Tumor size and body weight were measured during three weeks of treatment. Results: Significant tumor growth inhibition was observed, compared to the control, in the CDDP-treatment group, the $D O X$-treatment group, and the combination-treatment-group. Only the combination treatment regressed the tumor. Conclusion: $C D D P$ and DOX which are standard first-line therapies for osteosarcoma, may be clinically effective against primary osteosarcoma of the breast, and in particular, their combination.
\end{abstract}

Extraskeletal osteosarcomas are rare. Primary osteosarcoma of the breast is an exceedingly-rare malignant tumor with few cases

This article is freely accessible online.

Correspondence to: Robert M. Hoffman, Ph.D., AntiCancer Inc, 7917 Ostrow St, San Diego, CA, 92111, U.S.A. Tel: +1 8586542555, Fax: +18582684175, e-mail: all@anticancer.com

Key Words: Primary osteosarcoma of the breast, cisplatinum, doxorubicin, rare cancer, patient-derived orthotopic xenograft, nude mouse, efficacy. reported (1-3). Early recurrence and a tendency for hematogenous, but not lymphatic dissemination are common in such cases (4). Due to limited cases and data, there is currently no established first-line therapy for this disease. Clinical features of primary osteosarcoma of the breast have also yet to be defined $(1,4,5)$. The MAP regimen - high-dose methotrexate, doxorubicin, and cisplatinum - has improved the prognosis of patients with localized osteosarcoma (6). In the present study, part of this MAP regimen, standard-treatment against osteosarcoma, was tested for efficacy against primary osteosarcoma of the breast, which is histologically indistinguishable from non-soft tissue sarcomas $(2,4)$.

The first mouse model of primary osteosarcoma of the breast was established recently by our laboratory (4). In the present study, a patient-derived orthotopic xenograft (PDOX) model of osteosarcoma of the breast was used to test the efficacy of cisplatinum (CDDP) and doxorubicin (DOX) and their combination.

\section{Materials and Methods}

Mice. To establish a PDOX model of primary osteosarcoma of the breast, female athymic (nu/nu) nude mice (AntiCancer, Inc., San Diego, CA, USA) were used according to an AntiCancer, Inc. Institutional Animal Care and Use Committee (IACUC) protocol that was specifically approved for this study. The present study followed the principles and procedures outlined in the National Institutes of Health Guide for the Care and Use of Animals under Assurance Number A3873-1. All animal procedures have been previously described (7-14).

Patient-derived tumor. The surgically-resected primary osteosarcoma of the breast was obtained previously as part of a Kaiser Permanente San Diego Medical Center-approved protocol (4) (IRB\#12617).

Establishment of primary osteosarcoma of the breast PDOX nudemouse model. Four- $\mathrm{mm}^{3}$ tumor fragments were obtained from the first primary osteosarcoma of the breast patient-derived xenograft mouse model (4). To establish the PDOX model, nude mice were first anesthetized with isoflurane. A 5-mm skin incision was made 


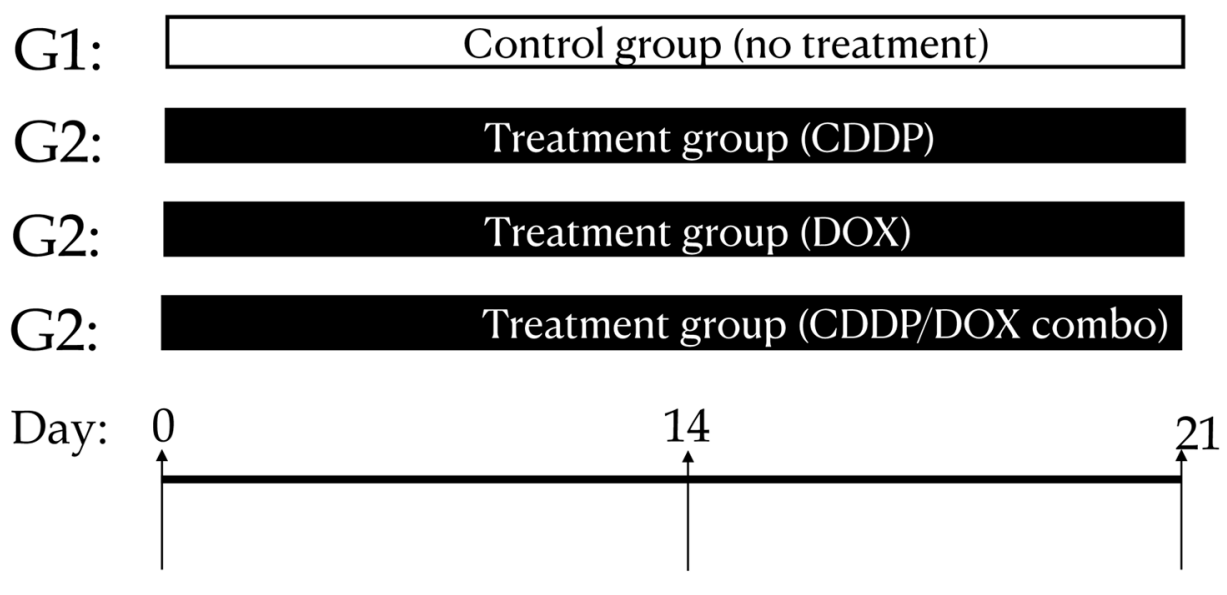

Sacrifice

Tumor and body-weight measurement: Days o, 14, 21

Treatment - cisplatinum (CDDP), doxorubicin (DOX) - administered: Days 0, 7, 14, 21

Figure 1. Schematic of treatment plan.

under the second nipple of the nude mice. A pocket was formed using surgical scissors by separating the skin from the dermis (4). Primary breast osteosarcoma fragments were then inserted into the pocket (4). The wound was closed with a 5-0 PDS-II suture.

Treatment scheme. The PDOX mouse models of primary osteosarcoma of the breast were randomly divided into four groups: control group; CDDP-treatment group; DOX-treatment group; and combination CDDP/DOX- treatment group with $\mathrm{n}=4$ for each group (Figure 1). After three weeks, tumor size was measured and treatment was initiated. For three weeks, the following treatment scheme was performed: control, no treatment; CDDP $(6 \mathrm{mg} / \mathrm{kg})$ by intraperitoneal injection, once a week; DOX (3 $\mathrm{mg} / \mathrm{kg})$ by intraperitoneal injection once a week; and combination-treatment (CDDP and DOX with the above doses) by intraperitoneal injection once a week. Mice were weighed and tumor size was measured with calipers. Tumor volume was calculated as previously described (8). All mice were sacrificed 6 weeks after implantation as previously described (8-14).

Statistical analysis. All statistical analyses were performed with JMP Pro ver. 15.0.0 (SAS Institute, Cary, NC, USA). A one-way ANOVA with a Tukey-Kramer multiple comparison test was used to compare groups. $p$-values were two-sided and a $p$-value of $\leq 0.05$ was regarded as statistically significant.

\section{Results}

Efficacy of CDDP and DOX on the primary osteosarcoma-ofthe-breast PDOX model. The treatment scheme is illustrated in Figure 1. CDDP alone and DOX alone inhibited tumor growth, and the combination of CDDP and DOX led to tumor regression. At day 21, the mean relative tumor volume ratio (tumor volume value on day 21 divided by the tumor volume on day 0 ) in the

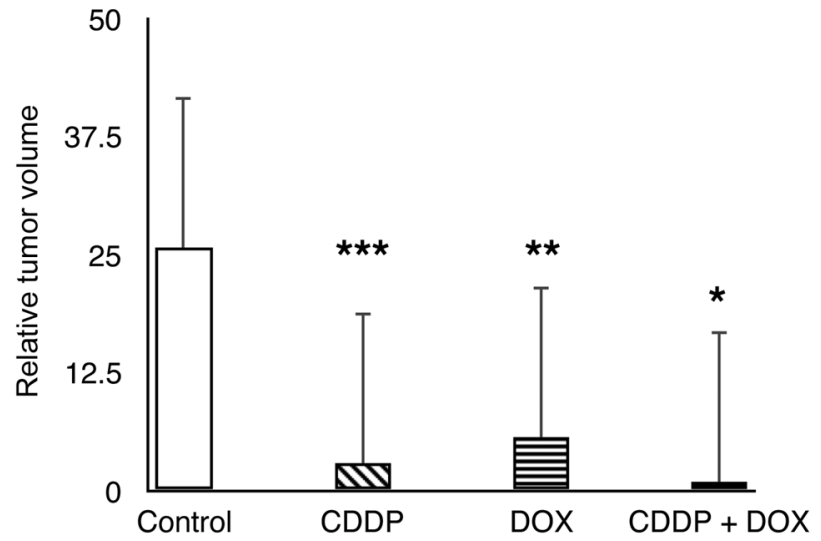

Figure 2. Treatment efficacy on relative tumor volume in primary osteosarcoma of the breast in a patient-derived orthotropic xenograft (PDOX) mouse model. $n=4$ mice for the control group and each treatment group. Control compared to the cisplatinum (CDDP)-treated mice $(* * * p=0.015)$; control compared to the doxorubicin $(D O X)$-treated mice $(* * p=0.032)$; control compared to the CDDP/DOX combinationtreated mice $(* p=0.0083)$.

control mice was 25.8; in the CDDP-treated mice was 2.90 $(p=0.015)$; in the DOX-treated mice was $5.66(p=0.032)$; and in the CDDP/DOX combination-treated mice was $0.90(p=0.0083)$, demonstrating regression of the tumor (Figure 2). Images of representative tumor-bearing mice demonstrated the efficacy of the therapies in the treatment groups with the CDDP/DOX combination being the most effective (Figure 3 ). The relative body weight of mice in the control group and treatment groups was not significantly different (Figure 4). 

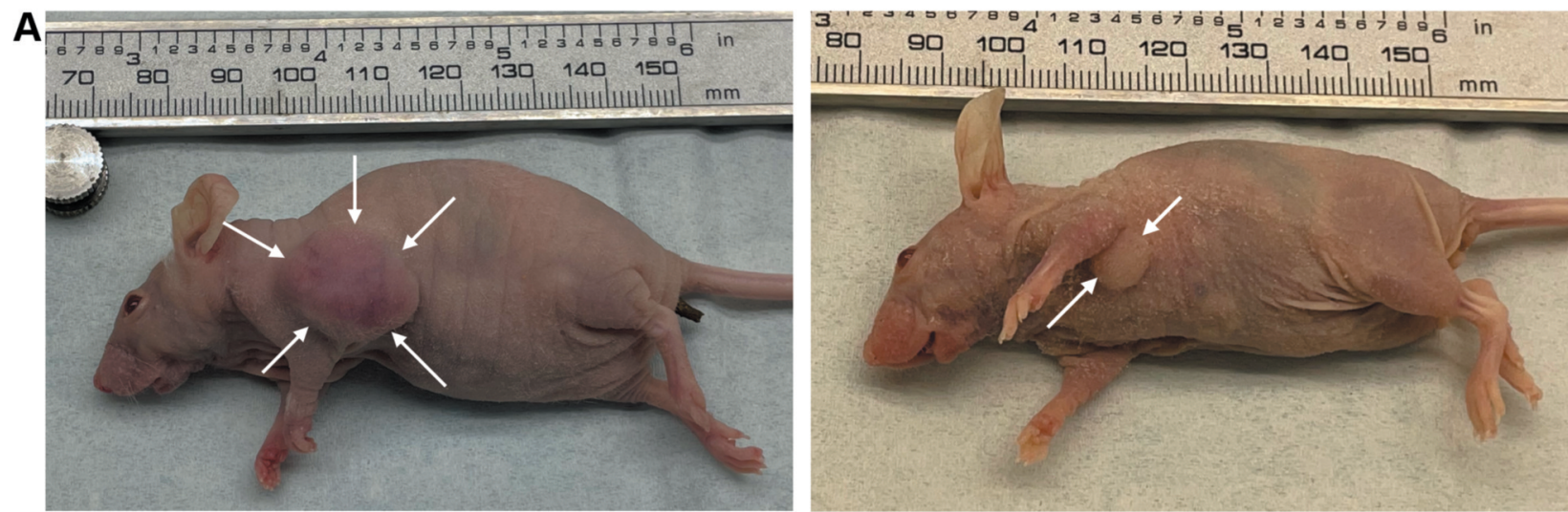

B
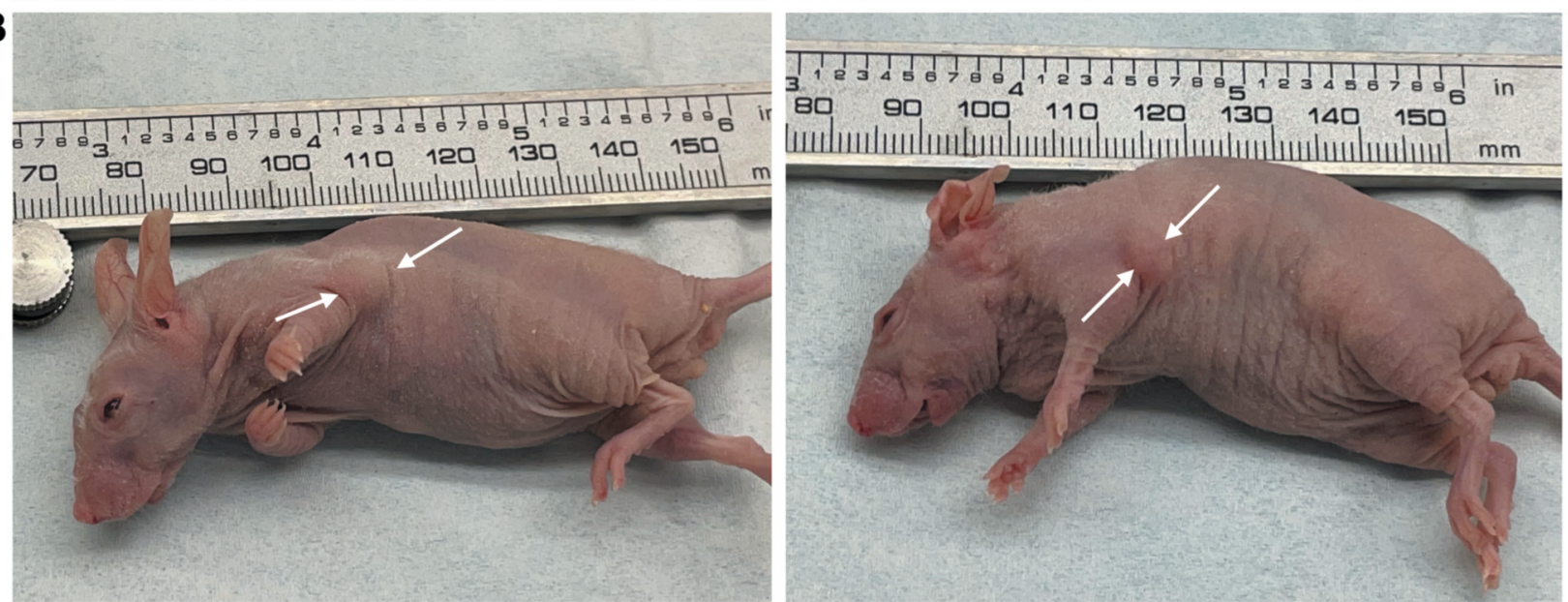

Figure 3. Primary osteosarcoma of the breast patient-derived orthotopic xenograft (PDOX) nude-mouse mouse model. (A) Mouse from the control group, day 21. (B) Mouse from the cisplatinum (CDDP)-treatment group, day 21. (C) Mouse from the doxorubicin (DOX)-treatment group, day 21. (D) Mouse from the CDDP/DOX-combination-treatment group, day 21.

\section{Discussion}

Primary osteosarcoma of the breast is an extremely-rare, malignant tumor. Using the first-established PDOX mouse model of this rare tumor developed in our laboratory (4), the present study determined that the combination of CDDP and DOX regressed the tumor. In the PDOX model, the tumor grows on the mammary gland of the mouse making it a clinically-relevant model in which candidates for clinical treatment can be evaluated. CDDP and DOX are currently first-line treatment of osteosarcoma of the bone. Therefore, these were the first candidates selected to determine their efficacy against primary osteosarcoma of the breast, which at present has no first-line therapy due to its extreme rarity. While treatment with each drug individually led to a statistically-significantly smaller tumor volume relative to the control group, only the combination of

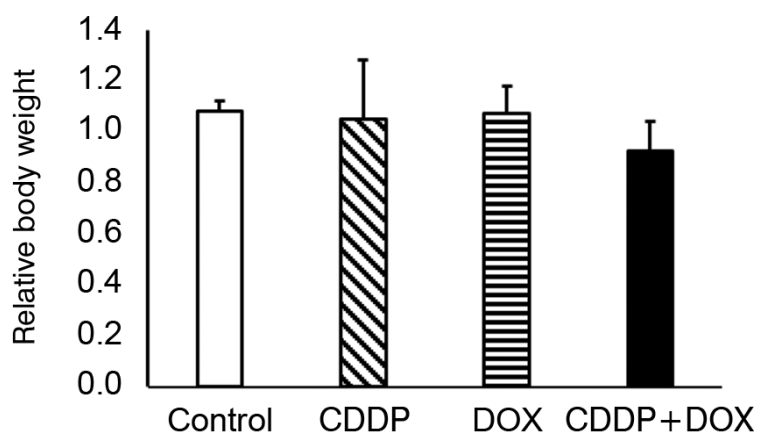

Figure 4. Relative body weight (body weight at the end of the experiment divided by body weight at the beginning of the experiment) of mice in patient-derived orthotopic xenograft mouse models of osteosarcoma of the breast. There was no significant difference in body weight between mice in the control group and treatment groups. 
both led to tumor regression with a tumor- volume ratio of less than one at the end of the treatment period. A tumor-volume ratio of less than one has potential for clinical efficacy. The present study suggests the combination of CDDP and DOX as a candidate clinical therapy for primary osteosarcoma of the breast, of which there is currently no established first-line therapy.

\section{Conflicts of Interest}

The Authors declare that there are no potential conflicts of interest regarding this study. AntiCancer Inc uses PDOX models for contract research. NFW, JY, YA, NM, CS and RMH are unsalaried affiliates of AntiCancer Inc.

\section{Authors' Contributions}

N.F.W., J.Y., and R.M.H designed and performed experiments and wrote the article; J.Y., Y.A., N.M., and C.S. provided technical support and conceptual advice. J.W. provided the surgically-resected tumor specimen. Writing, review, and/or revision of the manuscript: N.F.W., M.B., and R.M.H.

\section{Acknowledgements}

This paper is dedicated to the memory of A. R. Moossa, M.D., Sun Lee, M.D., Professor Li Jiaxi and Masaki Kitajima, M.D.

\section{Funding}

The present study was funded in part by the Robert M. Hoffman Foundation for Cancer Research, San Diego, CA, USA, which had no role in the design, execution, interpretation, or writing of the study.

\section{References}

1 Galama R, Matoso J, Capela G, Bôto C, Duarte C and Mendes AR: A rare etiology of a large tumoral mass of the breast - Case report primary leiomyosarcoma and osteosarcoma of the breast. Int J Surg Case Rep 78: 201-203, 2021 . PMID: 33360630. DOI: 10.1016/j.ijscr.2020.12.050

2 Bahrami A, Resetkova E, Ro JY, Ibañez JD and Ayala AG: Primary osteosarcoma of the breast: report of 2 cases. Arch Pathol Lab Med 131(5): 792-795, 2007. PMID: 17488168. DOI: 10.5858/2007-131-792-POOTBR

3 Silver SA and Tavassoli FA: Primary osteogenic sarcoma of the breast: a clinicopathologic analysis of 50 cases. Am J Surg Pathol 22(8): 925-933, 1998. PMID: 9706972. DOI: 10.1097/00000478-199808000-00002

4 Wu NF, Wu J, Yamamoto J, Aoki Y, Hozumi C, Bouvet M and Hoffman RM: The first mouse model of primary osteosarcoma of the breast. In Vivo 35(4): 1979-1983, 2021. PMID: 34182472. DOI: 10.21873 /invivo. 12466

5 Rizzi A, Soregaroli A, Zambelli C, Zorzi F, Mutti S, Codignola $\mathrm{C}$, Bertocchi P and Zaniboni A: Primary osteosarcoma of the breast: a case report. Case Rep Oncol Med 2013: 858705, 2013. PMID: 23691385. DOI: 10.1155/2013/858705

6 Isakoff MS, Bielack SS, Meltzer P and Gorlick R: Osteosarcoma: Current treatment and a collaborative pathway to success. J Clin Oncol 33(27): 3029-3035, 2015. PMID: 26304877. DOI: 10.1200/JCO.2014.59.4895
7 Higuchi T, Miyake K, Oshiro H, Sugisawa N, Yamamoto N, Hayashi K, Kimura H, Miwa S, Igarashi K, Chawla SP, Bouvet M, Singh SR, Tsuchiya H and Hoffman RM: Trabectedin and irinotecan combination regresses a cisplatinum-resistant osteosarcoma in a patient-derived orthotopic xenograft nudemouse model. Biochem Biophys Res Commun 513(2): 326-331, 2019. PMID: 30955860. DOI: 10.1016/j.bbrc.2019.03.191

8 Higuchi T, Oshiro H, Miyake K, Sugisawa N, Han Q, Tan Y, Park J, Zhang Z, Razmjooei S, Yamamoto N, Hayashi K, Kimura H, Miwa S, Igarashi K, Bouvet M, Chawla SP, Singh SR, Tsuchiya H and Hoffman RM: Oral recombinant methioninase, combined with oral caffeine and injected cisplatinum, overcome cisplatinumresistance and regresses patient-derived orthotopic xenograft model of osteosarcoma. Anticancer Res 39(9): 4653-4657, 2019. PMID: 31519563. DOI: 10.21873/anticanres.13646

9 Higuchi T, Sugisawa N, Miyake K, Oshiro H, Yamamoto N, Hayashi K, Kimura H, Miwa S, Igarashi K, Bouvet M, Singh SR, Tsuchiya $\mathrm{H}$ and Hoffman RM: The combination of olaratumab with doxorubicin and cisplatinum regresses a chemotherapyresistant osteosarcoma in a patient-derived orthotopic xenograft mouse model. Transl Oncol 12(9): 1257-1263, 2019. PMID: 31299622. DOI: 10.1016/j.tranon.2019.06.002

10 Higuchi T, Sugisawa N, Miyake K, Oshiro H, Yamamoto N, Hayashi K, Kimura H, Miwa S, Igarashi K, Chawla SP, Bouvet M, Singh SR, Tsuchiya H and Hoffman RM: Sorafenib and palbociclib combination regresses a cisplatinum-resistant osteosarcoma in a PDOX mouse model. Anticancer Res 39(8): 4079-4084, 2019. PMID: 31366491. DOI: 10.21873/anticanres.13565

11 Higuchi T, Sugisawa N, Miyake K, Oshiro H, Yamamoto N, Hayashi K, Kimura H, Miwa S, Igarashi K, Kline Z, Belt P, Chawla SP, Bouvet M, Singh SR, Tsuchiya H and Hoffman RM: Combination treatment with sorafenib and everolimus regresses a doxorubicin-resistant osteosarcoma in a PDOX mouse model. Anticancer Res 39(9): 4781-4786, 2019. PMID: 31519579. DOI: 10.21873/anticanres.13662

12 Higuchi T, Sugisawa N, Miyake K, Oshiro H, Yamamoto N, Hayashi K, Kimura H, Miwa S, Igarashi K, Kline Z, Bouvet M, Singh SR, Tsuchiya H and Hoffman RM: Pioglitazone, an agonist of PPAR $\gamma$, reverses doxorubicin-resistance in an osteosarcoma patient-derived orthotopic xenograft model by downregulating Pglycoprotein expression. Biomed Pharmacother 118: 109356, 2019. PMID: 31545293. DOI: 10.1016/j.biopha.2019.109356

13 Higuchi T, Sugisawa N, Yamamoto J, Oshiro H, Han Q, Yamamoto N, Hayashi K, Kimura H, Miwa S, Igarashi K, Tan Y, Kuchipudi S, Bouvet M, Singh SR, Tsuchiya H and Hoffman RM: The combination of oral-recombinant methioninase and azacitidine arrests a chemotherapy-resistant osteosarcoma patient-derived orthotopic xenograft mouse model. Cancer Chemother Pharmacol 85(2): 285-291, 2020. PMID: 31705268. DOI: $10.1007 / \mathrm{s} 00280-019-03986-0$

14 Higuchi T, Yamamoto J, Sugisawa N, Tashiro Y, Nishino H, Yamamoto N, Hayashi K, Kimura H, Miwa S, Igarashi K, Bouvet M, Singh SR, Tsuchiya H and Hoffman RM: PPAR $\gamma$ agonist pioglitazone in combination with cisplatinum arrests a chemotherapy-resistant osteosarcoma PDOX model. Cancer Genomics Proteomics 17(1): 35-40, 2020. PMID: 31882549. DOI: $10.21873 / \operatorname{cgp} .20165$

Received August 24, 2021

Revised September 7, 2021

Accepted September 8, 2021 